\title{
Vorwort
}

\section{Robert Galler}

Lehrstuhl für Subsurface Engineering, ZAB - Zentrum am Berg, Montanuniversität Leoben, Leoben, Österreich

Online publiziert 4. Dezember 2017

Liebe Geotechniker und Freunde des konstruktiven Tiefbaus!

Es ist wieder einmal so weit, dass wir geotechnische Fragenstellungen im Fokus dieser Ausgabe der BHM haben. Wir beginnen mit einem Beitrag zu Steinschlagsimulationen zur Abschätzung von Gefahrenbereichen in Tagebauen, wo es programmtechnisch neue Entwicklungen gibt. Im Anschluss daran folgt ein Beitrag, der aufzeigen soll, wie geotechnisches Monitoring zur Maßnahmenentwicklung und Projektumsetzung der Sanierung der Großrutschung Weißengriesgraben in Bad Aussee herangezogen werden konnte und damit der Sicherheit der Bahntrasse im betroffenen Bereich diente.

Die Lehrstühle für Bergbaukunde, Bergtechnik und Bergwirtschaft sowie Subsurface Engineering - Geotechnik und Tunnelbau verfügen am Standort Leoben gemeinsam über ein modernst ausgestattetes Labor zur Charakterisierung von Geomaterialien und Baustoffen, sei es in Kurzzeit- oder auch Langzeitversuchen. Wir haben uns zum Ziel gesetzt, dies mit einem Beitrag in der aktuellen Ausgabe der BHM darzulegen.

Darauf aufbauend wollen wir mit einem weiteren Beitrag zu diesem Thema nachweisen, wie wichtig in vielen Fällen die Erfassung von Eingabeparametern aus erweiterten Laborversuchen zur Erzielung realistischer Ergebnisse aus numerischen Simulationen ist, insbesondere wenn dort höherwertige Materialgesetze verwendet werden müssen und das Materialverhalten im Post-Failure Bereich dargestellt werden soll.

Ein weiterer Beitrag setzt sich mit den während der Tunnelvortriebsarbeiten entlang der S35 angetroffenen Serpentiniten auseinander. Dabei wurden antigoritische Serpentinitproben im Detail untersucht und es konnte gezeigt werden, dass die beobachteten hohen Silikatfasergehalte in der Tunnelluft zu einem überwiegenden Anteil auf asbestiformen Antigorit zurückzuführen sind, wobei Chry-

R. Galler ( $\triangle)$

Lehrstuhl für Subsurface Engineering, ZAB - Zentrum am Berg, Montanuniversität Leoben,

8700 Leoben, Österreich

Robert.Galler@unileoben.ac.at sotil- und Antigoritfasern mit der Raster-Elektronenmikroskopie kombiniert mit energie-dispersiver Spektroskopie nicht sicher unterschieden werden können.

Zuguterletzt berichten wir über eine aktuelle Entwicklung im maschinellen Tunnelbau; konkret über ein Kamerasystem für die geologische Dokumentation vor dem Bohrkopf einer Vollschnitttunnelbohrmaschine. Das Kamerasystem wird aktuell auf einer TBM des Bauloses Tulfes - Pfons des Brenner-Basistunnels getestet. Es liefert hochauflösende Bilder und eine auf Fotogrammetrie basierende vollflächige 3D-Dokumentation, indem es in Vortriebspausen in Diskenkästen im Bohrkopf montiert und die gesamte Ortsbrust bis in den Kaliberbereich befahren wird.

Abschließend darf ich berichten, dass die Vortriebsarbeiten am ZaB - Zentrum am Berg voll im Gange sind und wir aus heutiger Sicht davon ausgehen, dass wir diese bis Ende 2018 abschließen können werden. Über die diesbezüglichen Arbeiten wollen wir aber erst im Heft 12 der BHM 2018 berichten.

Ich wünsche Ihnen eine frohe Weihnachtszeit und alles Gute für das neue Jahr 2018!

Ein herzliches Glückauf!

Robert Galler

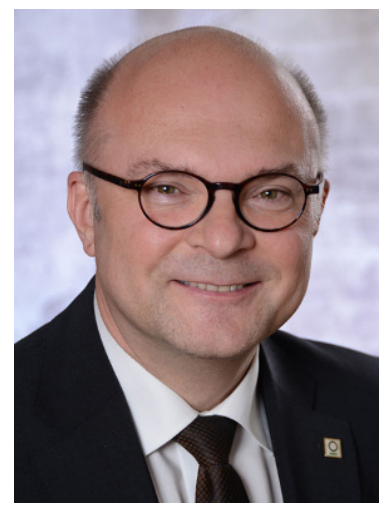

Robert Galler 